\title{
Hydroxytyrosol But Not Resveratrol Ingestion Induced an Acute Increment of Post Exercise Blood Flow in Brachial Artery
}

\author{
Giorgia Sarais ${ }^{1}$, Antonio Crisafulli², Daniele Concu ${ }^{3}$, Andrea Foiss ${ }^{4}$, Abdallah Raweh ${ }^{5}$, Alberto Concu ${ }^{3,5}$ \\ ${ }^{1}$ Department of Life and Environmental Sciences, University of Cagliari, Cagliari, Italy \\ ${ }^{2}$ Laboratory of Sports Physiology, University of Cagliari, Cagliari, Italy \\ ${ }^{3}$ IIC Technologies Ltd., Cagliari, Italy \\ ${ }^{4}$ EventFeel Ltd., Cagliari, Italy \\ ${ }^{5}$ Medical Sciences Faculty, The LUdeS Foundation Higher Education Institution, Kalkara, Malta \\ Email: aconcu44@gmail.com
}

How to cite this paper: Sarais, G., Crisafulli, A., Concu, D., Fois, A., Raweh, A. and Concu, A. (2016) Hydroxytyrosol But Not Resveratrol Ingestion Induced an Acute Increment of Post Exercise Blood Flow in Brachial Artery. Health, 8, 1766-1777. http://dx.doi.org/10.4236/health.2016.815170

Received: August 19, 2016

Accepted: December 11, 2016

Published: December 14, 2016

Copyright $\odot 2016$ by authors and Scientific Research Publishing Inc. This work is licensed under the Creative Commons Attribution International License (CC BY 4.0).

http://creativecommons.org/licenses/by/4.0/ (c) (i) Open Access

\begin{abstract}
The aim of this study was to test if previous ingestion of compounds containing resveratrol or hydroxytyrosol, followed by an exhausting hand grip exercise, could induce an acute post-exercise increase in brachial blood flow. Six healthy subjects (three males and three females, $35 \pm 7$ years), 60 minutes after ingestion of a capsule containing $200 \mathrm{mg}$ of resveratrol or $30 \mathrm{ml}$ of extra virgin olive oil enriched with tyrosol, oleuropein and hydroxytyrosol, performed a hand grip exercise equal to half of their maximum strength until they were no longer able to express the same force (2-day interval between tests). The nonparametric Wilcoxon signed rank test was used for statistical evaluations. Brachial artery blood flow (Fba) and both blood velocity (Vba) and artery diameter ( $\mathrm{Dba}$ ) were assessed immediately after exercise cessation by means of colour Doppler ultrasound. After ingestion of the oil mixture the post-exercise value of Fba median was 2.4 times higher than that after ingestion of the resveratrol compound $(\mathrm{P}=0.03)$, and also the Vba median concerning the hydroxytyrosol was 1.9 times higher than that of the resveratrol $(P=0.03)$. Both functional foods did not lead to a significant difference in the Dba medians. These results indicate that hydroxytyrosol, but not resveratrol, may be an effective adjuvant of recreational or agonistic, long-lasting sports performances, thanks to the powerful blood flow increment which can be obtained as soon as one hour from its oral intake.
\end{abstract}

\section{Keywords}

Functional Foods, Hydroxytyrosol, Resveratrol, Hand Grip, Blood Flow 


\section{Introduction}

Both resveratrol from peel grapes and hydroxytyrosol from olive leaves are considered functional foods since they are powerful antioxidants [1] [2] [3] [4]. However, there is increasing evidence for their role also on the endothelial function of the vessels. An in vitro study of Leikert et al. [5] used human umbilical vein endothelial cells treated with increasing concentrations of a polyphenolic extract of red wine and kept in incubation for 20 hours, and they showed that cellular production of nitrogen monoxide (NO) increased due to doubling of the levels of the enzyme nitric oxide synthase (eNOS). From these results, although in vitro, it can be assumed that the resveratrol content of red wine extract may induce an increase in NO in these endothelial cells from which a smooth muscle relaxation of the tunica media of these vessels, thus their dilation, could be expected in vivo [6] [7]. Reyes et al. [8] studied in vitro the effects of hydroxytyrosol in human blood samples and they found a concentration-dependent effect of this polyphenol in inhibiting platelet aggregation due to an increase in NO production. Storniolo et al. [9] in an in vitro model that simulates the condition of type 2 diabetes showed a reverted glucose inducing decrease in endothelial eNOS phosphorylation by hydroxytyrosol extract from extra virgin olive oil. These latter experimental results also suggest a possible vasodilator effect of hydroxytyrosol. Nowadays it has been observed that the shear stress on the endothelium of blood vessels due to circulating blood is a powerful stimulus for NO release [10]. Thus the chronic increase in endothelial shear stress induced by the exercise also induces higher levels of gene coding for endothelial eNOS expression, determining in this way an increase in endothelium-dependent muscle relaxation [11]. This occurrence may derive a fall in the vascular resistance of a given vascular district. However, several studies have shown that acute exercise can also have a not negligible impact on endothelial function [12] [13] [14] [15]. Indeed, a review by Padilla et al. [16] indicates that acute exercise can be utilized as a model to allow for modulating more efficiently the mode, duration and intensity of exercise on the basis of the endothelial response. In these experiments we wanted to see if the intake of resveratrol or hydroxytyrosol before an acute and exhaustive hand grip exercise could induce a post-exercise blood flow increase in a given vascular district of trained subjects.

\section{Materials and Methods}

\subsection{Subjects}

To carry out the experiments with a group of relatively homogenous subjects among graduates in medicine and surgery who were attending postgraduate school in sports medicine at the University of Cagliari (Italy), we found healthy volunteers who were moderately trained. The admission criteria were: no chronic degenerative disease of the cardiovascular and respiratory apparatus, of the nervous-endocrine-metabolic system and of the muscle-skeletal apparatus. Exclusion criteria were: current smoking, the condition of physical inactivity, taking medications, habitual drinking of alcohol, subclinical overweight and intolerance to glucose. Eighteen volunteers responded to our 
call, each of whom was subjected to a thorough investigation of medical history and to an examination by a sports medicine specialist with detection, at rest, of the ECG, heart rate (R-HR) and mean arterial blood pressure (R-MBP) together with the body mass index (BMI) obtained by dividing body weight by height squared. After these medical controls only six subjects, three males and three females, presented the required characteristics and were engaged in these experiments. Table 1 shows the socio- demographic and clinical characteristics of these subjects.

\subsection{Instrumentation}

The device used for the experiments was a hand grip dynamometer (Kern, USA), with settable springs. It displays in real time the produced strength, the maximum strength of a subject's grip and the average strength of several grips.

To assess in a non-invasive way the blood flow in a brachial artery, we employed a widely used technique [17] based on the measurement of the velocity of blood in the vessel, obtained with the ultrasonic Doppler method, and the measurement of the cross-section in the same blood vessel obtained with the ultrasound method. In these experiments, to measure the blood flow in the brachial artery of the hand that performed the exercise, a colour Doppler echocardiograph (M5 Diagnostic Ultrasound System, Mindray Bio-Medical Electronics Co., Shenzhen, China) was utilized with an ultrasound probe type 7L4 suitable for arterial flow measures. The ultrasound probe was positioned in the bend of the elbow at a $60^{\circ}$ angle with respect to the plane in which the brachial artery lay.

\subsection{Experimental Procedures}

The experimental sessions were held at $3 \mathrm{pm}$ at the laboratory of sports physiology of the Department of Medical Sciences, University of Cagliari, Italy. The temperature was stabilized between $20^{\circ} \mathrm{C}$ and $22^{\circ} \mathrm{C}$.

The hand grip exercise consisted of contracting and relaxing the fingers of one hand

Table 1. For each engaged subject are represented the anthropometric variables (age, weight, height and BMI) together with the recreational activities quantified in terms of hours per week and the rest values of heart rate (R-HR) and mean arterial blood pressure (R-MBP).

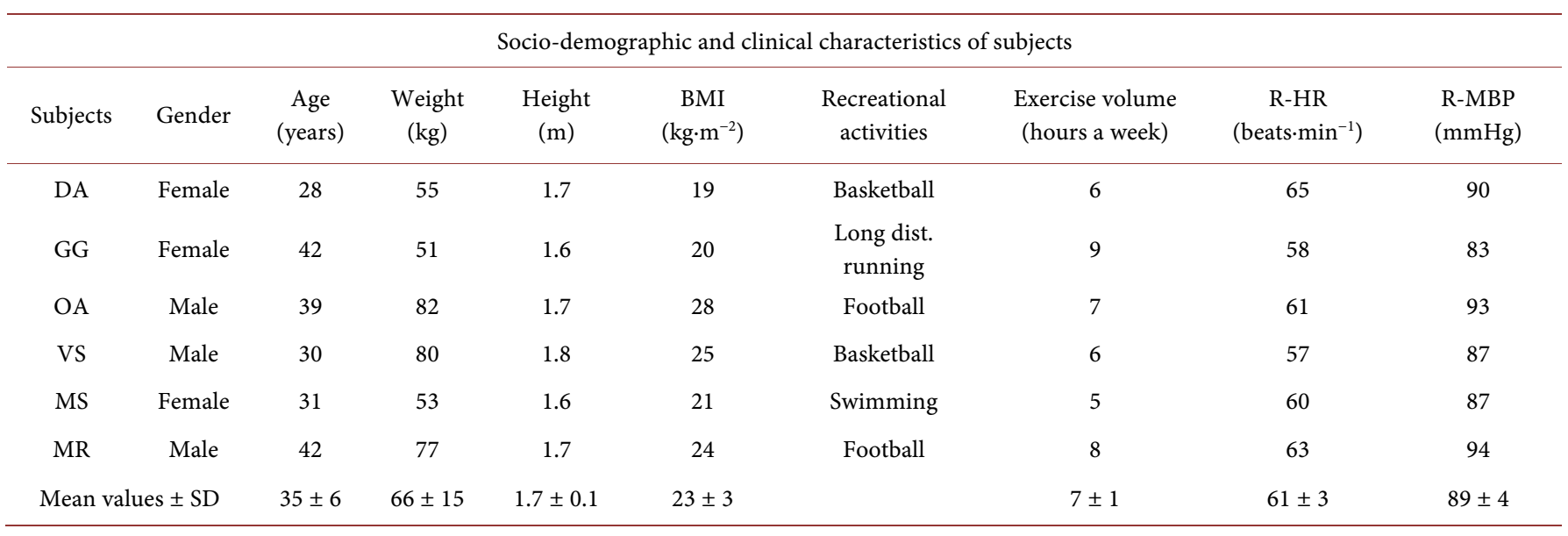


against a spring resistance equal to half of the maximum force, in $\mathrm{kg}$, expressed with this gesture. The contraction-relaxation cycle of the fingers lasted 2 seconds and was repeated until the subject was no longer able to express the same force. In Figure 1 are represented mean values of the maximum load developed in a preliminary session of hand gripping in which subjects were invited to release their highest hand contraction, and of the constant load developed throughout the sessions in which the exercise lasted up to exhaustion.

Figure 2 shows one subject while performing a hand grip exercise with the right hand. He was seated with his elbow resting on the surgery cot of the laboratory of sports physiology. Also shown is the ultrasound probe while positioning it in the inner bend of the elbow, being careful to position it at an angle of about $60^{\circ}$ with respect to the brachial artery direction.

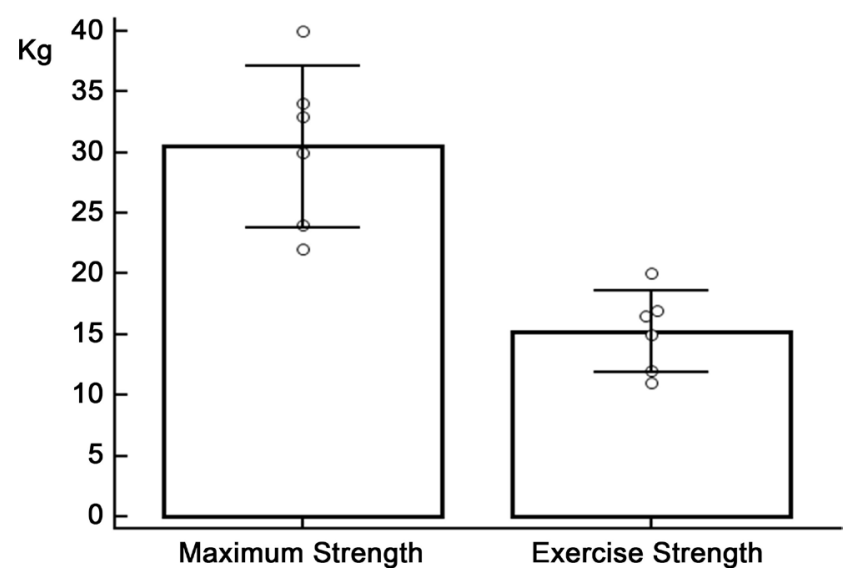

Figure 1. The column on the left represents the mean value, expressed in $\mathrm{kg}$, of the maximum strength delivered by the subjects against the dynamometer by finger contraction; the column on the right corresponds to half the maximum strength that was maintained up to exhaustion. Vertical bars represent the \pm standard deviation while circles along them, respectively for maximum and exercise strengths, show the distribution of each participant.
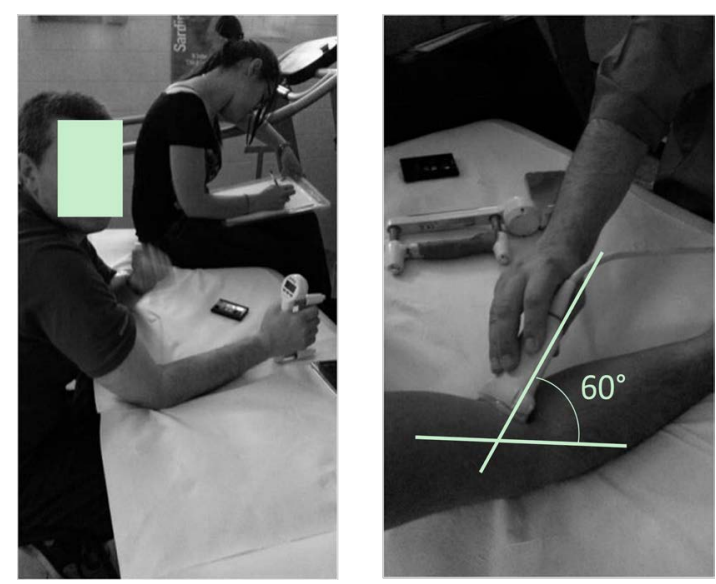

Figure 2. The image on the left shows a subject engaged in the hand grip exercise up to exhaustion. The image on the right shows how the ultrasound probe was positioned in the bend of the subject's elbow at a $60^{\circ}$ angle with respect to the plane of the brachial artery. 


\subsection{Experimental Protocol}

The experimental protocol consisted of each subject performing the following tests:

i) Base at Rest (BR) - which consisted of assessing the brachial artery blood flow (Fba) together with Arterial blood velocity (Vba) and arterial diameter (Dba) with the ultrasound probe while a subject remained seated at rest;

ii) Exercise plus Resveratrol (EXresv)-where subjects assumed orally one capsule containing $200 \mathrm{mg}$ of resveratrol extracted from the roots of Polygonum cuspidatum (Resveratrox, produced by Solgar, Italy); they then remained seated at rest for $60 \mathrm{~min}$ before performing a hand grip exercise up to exhaustion, after which the Fba, Vba and Dba in the arm that had worked was again assessed;

iii) Exercise plus Hydroxytyrosol (EXhydr)—where subjects assumed orally $30 \mathrm{ml}$ of extra virgin olive oil enriched with tyrosol $(18.8 \mathrm{mg} / \mathrm{kg})$, oleuropein $(101.3 \mathrm{mg} / \mathrm{kg})$ and hydroxytyrosol $(19.0 \mathrm{mg} / \mathrm{kg})$; they then remained seated at rest for $60 \mathrm{~min}$ before performing a hand grip exercise up to exhaustion, after which the Fba, Vba and Dba in the arm that had worked was again assessed. The oil mixture was prepared by the pharmaceutical laboratory of the Department of Life and Environment Sciences, University of Cagliari, Italy, by extracting these added substances from olive leaves.

Subjects were randomly submitted to two experimental sessions with a 2-day interval between them. In session A they performed a BR test after which they remained seated at rest for $60 \mathrm{~min}$; they then performed the EXresv test. In session B they performed the same tests as previously described, but instead of test EXresv they performed the EXhydr test.

Those participating in the experiments were unaware of the composition of the substances ingested during sessions A and B, nor did they know the experimental hypothesis on which the study was based.

Finally, when both A and B sessions were stopped, we asked to each participant to tell us about possible differences in the perceived sensations during each of these two tests.

\subsection{Data Analysis}

Due to the asymmetric distribution of the data, the visualization of the assessed values of the hemodynamic variables was made by means of the box and whiskers plots [18] in which we have also included the spread of data. Each box ranged from the $1^{\text {st }}$ quartile (Q1) to the $3^{\text {rd }}$ quartile $(\mathrm{Q} 3)$ of the distribution, so the box length is the interquartile range (IQR), which contains the central $50 \%$ of data, and the line across the box indicates the statistical median, or Q2. The whiskers are lines extending from $1^{\text {st }}$ and $3^{\text {rd }}$ quartile, respectively, to the most extreme data points within Q1 $-1.5 \times \mathrm{IQR}$ and Q3 + $1.5 \times$ IQR [19]. Moreover, due to the modest sample size of our data, when comparing the Fba, Vba and Dba post-exercise values between the resveratrol and hydroxytyrosol groups of data, we have chosen the nonparametric Wilcoxon signed rank test. The same test was also applied to compare the differences of the "delta" $(\Delta)$ values between the two groups of data as (EXresv - BRresv) versus (EXhydr - BRhydr). In all the compar- 
isons the statistical significance was established as a $\mathrm{P}$ value $<0.05$. Statistical tests were carried out utilizing commercially available software (MedCalc, Belgium).

\section{Results}

After experimental sessions were finished, no subject reported sensible differences in perceiving sensation from the hand grip exercise when resveratrol or hydroxytyrol had been ingested before beginning the exercise.

Concerning the test duration, in session $A$ the mean \pm SE value of the EXresv test was $276 \pm 72 \mathrm{~s}$ while in session B the mean \pm SE value of the EXhydr test was $279 \pm 71$ s. Comparing these mean duration it was found that the difference between them was not statistically significant. In Figure 3 the box concerning BRresv values of $\mathrm{Fba}$ is smaller than that concerning the BRhydr ones. In fact, the BRresv showed an IQR from 73.2 to $196.8 \mathrm{ml} \cdot \mathrm{min}^{-1}$ while the BRhydr IQR was from 89.6 to $369.5 \mathrm{ml} \cdot \mathrm{min}^{-1}$. However, their respective median lines are very close $\left(\right.$ BRresv $=142.9 \mathrm{ml} \cdot \mathrm{min}^{-1}$ and $\mathrm{BRhydr}=$ $125.9 \mathrm{ml} \cdot \mathrm{min}^{-1}$ ) with no statistically significant difference between them. The same figure clearly shows that the box of EXhydr data (median $=516.1 \mathrm{ml} \cdot \mathrm{min}^{-1}$ with IQR from 374.3 to $786.18 \mathrm{ml} \cdot \mathrm{min}^{-1}$ ) is placed totally above the box of EXresv data (median = $211.6 \mathrm{ml} \cdot \mathrm{min}^{-1}$ with IQR from 108.2 to $330.8 \mathrm{ml} \cdot \mathrm{min}^{-1}$ ), and the statistical comparison between these two groups of data showed a $\mathrm{P}=0.03$. When Fba $\Delta$ was also calculated, the median concerning the hydrixytyrosol group was of $404.5 \mathrm{ml} \cdot \mathrm{min}^{-1}$ while the median concerning the resveratrol group was of $92.1 \mathrm{ml} \cdot \mathrm{min}^{-1}$. The comparison between these two groups of data comes very close to statistical significance $(P=0.06)$.

Figure 4 concerns the Vba data and shows that the BRresv box data (median $=20.3$ $\mathrm{cm} \cdot \mathrm{s}^{-1}$ with IQR from 17.6 to $24.9 \mathrm{~cm} \cdot \mathrm{s}^{-1}$ ) could be entirely contained inside the BRhydr

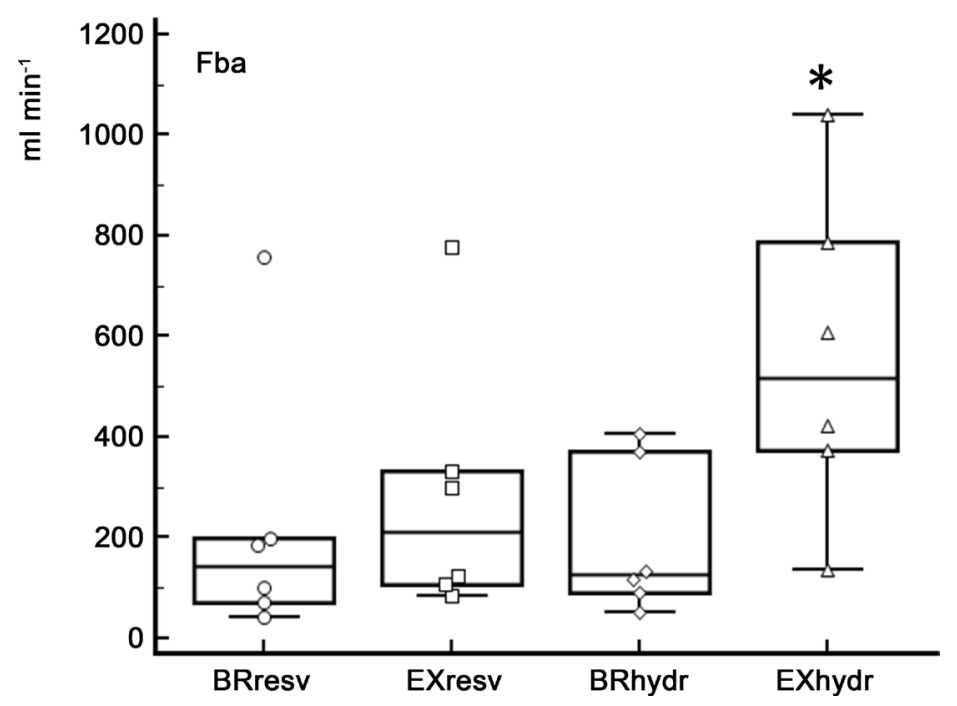

Figure 3. The experimental data concerning the blood flow values in the brachial artery (Fba) in the different experimental conditions are represented as box and whiskers diagrams with markers that reveal the distribution of each participant. Outlier marks are shown outside the whiskers from $1^{\text {st }}$ quartiles of BRresv and EXresv plots. $\left({ }^{*}\right)$ With respect to EXresv: $\mathrm{P}=0.03$. 


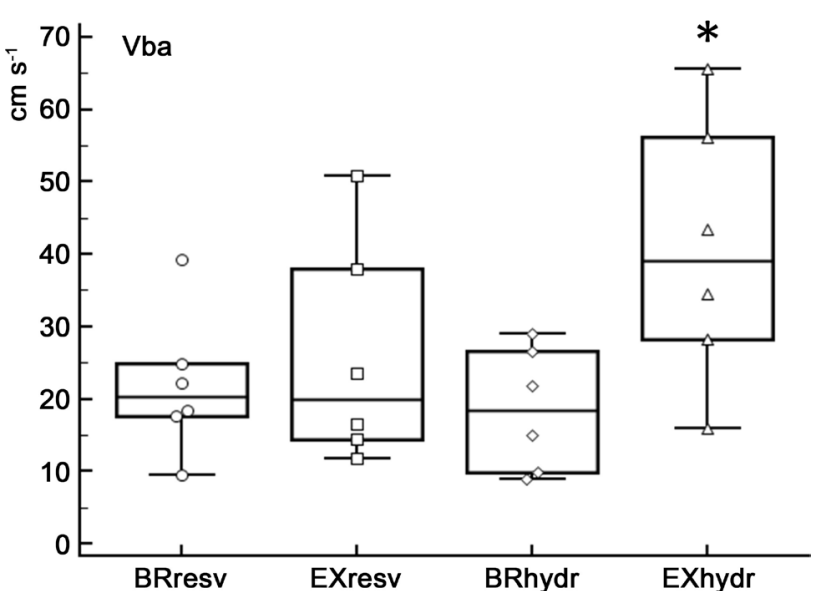

Figure 4. The experimental data concerning the blood velocity values in the brachial artery (Vba) in the different experimental conditions are represented as box and whiskers diagrams with markers that reveal the distribution of each participant. An outlier mark is shown outside the whisker from $1^{\text {st }}$ quartile of BRresv plot. $\left(^{*}\right)$ With respect to EXresv: $\mathrm{P}=0.03$.

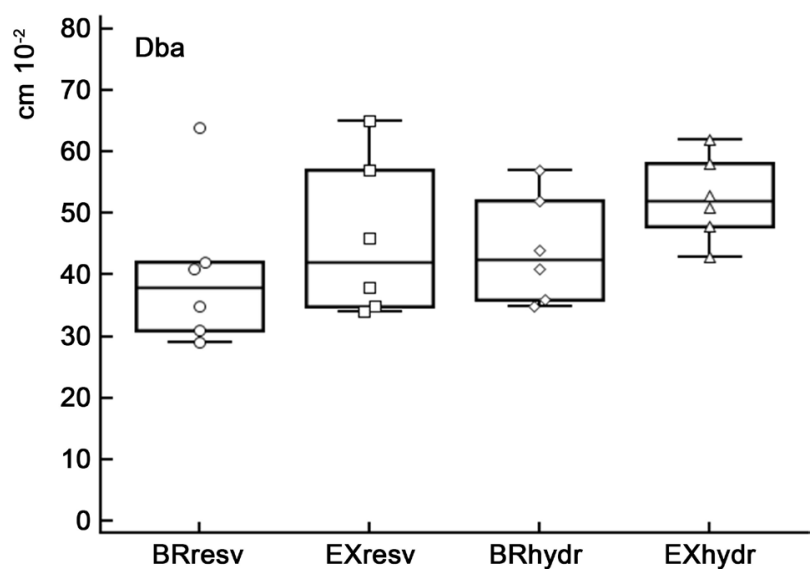

Figure 5. The experimental data concerning the diameter values of the brachial artery (Dba) in the different experimental conditions are represented as box and whiskers diagrams with markers that reveal the distribution of each participant. An outlier mark is shown outside the whisker from $1^{\text {st }}$ quartile of BRresv plot.

box (median $=22.9 \mathrm{~cm} \cdot \mathrm{s}^{-1}$ with IQR from 16.4 to $28.4 \mathrm{~cm} \cdot \mathrm{s}^{-1}$ ). So the comparison between these two groups of data did not reach any statistically significant difference. The figure also shows that despite a practically complete overlap between Q3 of the EXhydr box (IQR: from 28.3 to $56.1 \mathrm{~cm} \cdot \mathrm{s}^{-1}$ ) and Q1 of the EXresv box (IQR: from 14.4 to 37.9 $\left.\mathrm{cm} \cdot \mathrm{s}^{-1}\right)$, the median in the former box $\left(39.1 \mathrm{~cm} \cdot \mathrm{s}^{-1}\right)$ was almost doubled compared with that of the second box $\left(20.1 \mathrm{~cm} \cdot \mathrm{s}^{-1}\right)$. These differences between EXhydr and EXresv data were statistically significant $(P=0.03)$. The comparison between Vbe $\Delta$ values showed that the median of the hydroxytyrosol group was of $19.7 \mathrm{~cm} \cdot \mathrm{s}^{-1}$ while that of the resveratrol one was of $6.9 \mathrm{~cm} \cdot \mathrm{s}^{-1}$, or nearly three times lower than that concerning hydroxytyrosol, and the comparison between these $\Delta$ values reached the statistical significance $(\mathrm{P}=0.03)$.

Box and whiskers plots represented in Figure 5 show that Dba values in BRresv (me- 
dian $=0.38 \mathrm{~cm}$ with IQR from 0.31 to $0.42 \mathrm{~cm}$ ) and BRhydr (median $=0.42 \mathrm{~cm}$ with IQR from 0.36 to $0.52 \mathrm{~cm}$ ), were quite scattered and their comparison did not reach a significant difference. The same behaviour can be observed when considering post- exercise Dba measurements. In fact, Dba median in the EXhydr box was $0.52 \mathrm{~cm}$ with IQR from 0.48 to $0.58 \mathrm{~cm}$, and the same variable in the EXresv box was $0.42 \mathrm{~cm}$ with IQR from 0.35 to $0.57 \mathrm{~cm}$. Post-exercise Dba data comparison did not reach the significant difference. As expected, also Dba $\Delta$ values between post-exercise versus base at rest differences for hydroxytirosol $($ median $=0.04 \mathrm{~cm}$ ) did not differ significantly from the same $\Delta$ values for resveratrol $($ median $=0.02 \mathrm{~cm})$.

\section{Discussion}

The main result from these experiments is the evidence that the pre-exercise ingestion, by moderately trained young subjects, of a hydroxytyrosol enriched extra virgin olive oil, induced a large blood flow post-exercise increase in the brachial artery of the arm that had performed an exhaustive hand grip exercise, mainly due to blood speed increase. A second important result, parallel to the first, is that when the same subjects repeated the hand grip protocol, but with the pre-exercise ingestion of an adequate dose of resveratrol, no increase was found in the blood flow of the brachial artery. Thus, despite these two nutraceutical compounds both being known for playing an important role in the relationships between vessels and blood, only the hydroxytyrosol showed to possess a marked post-exercise activity in increasing the speed of blood flow of the brachial arteries in a small but homogeneous group of trained subjects.

The effect of the hydroxytyrosol in increasing the post-exercise Fba, obtained in the present experiments, agrees with the results of a recent paper of Valls et al. [20] in which hypertensive patients who ingested $30 \mathrm{ml}$ of extra virgin olive oil enriched with phenolic compounds containing hydroxytyrosol, were submitted to a brachial ischemic reactive hyperaemia. Five hours after ingestion of the enriched oil, it was found that the reactive hyperaemia had significantly increased with respect to a test in which ischemia was applied with ingestion of non-enriched oil. Since it is known that the test to induce a reactive hyperaemia induces vasodilation [10], the above experiment, which utilized an oil mixture that was qualitatively similar to that used in the present experiment, indirectly reinforces our results of a possible influence of the oil mixtures containing hydroxytyrosol on the modulators of blood flow in the brachial artery. Concerning resveratrol, Wong et al. [21] performed a test similar to that of Valls et al. [20] in obese individuals after they acutely ingested $270 \mathrm{mg}$ of resveratrol and, compared to a placebo, the flow-mediated dilation of the brachial artery had significantly increased. To explain this experimental divergence between our results and those of Wong et al., it is to be considered that our subjects ingested $200 \mathrm{mg}$ of resveratrol which may be an insufficient dose to induce significant increases in Dba, and then in Fba. Nevertheless, Posadino et al. [22] recently showed that, in endothelial cells, tissue-attainable doses of resveratrol increased the intracellular oxidative state, affecting in this way mitochondrial membrane depolarization which induces death in these cells. Thus, care must be taken 
concerning resveratrol dosage. Although the number of participants in these experiments was relatively small, Table 1 shows that their socio-demographic characteristics can be considered sufficiently homogeneous, so as to ensure a good model for the study of the mechanisms that are the subject of this research. In fact, participants have the same socio-cultural background and all are healthy and relatively young, and all of them perform weekly a considerable and similar amount of physical exercise. Moreover, sports in which they are engaged are characterized by alternating recruitment of energy from aerobic and anaerobic substrates and, in addition, as suggested by the HR and MBP values at rest, they implemented the typical functional adaptations of trained people. Despite this, one could argue that, probably, changes in their vessel endothelial function induced by exercise may differ in male and female subjects [23], thus our mixed experimental group may result in distorted data due to this demographic condition. However, several previous studies [24] [25] [26] [27] have shown that gender differences do not lead to serious differences in the endothelial response to specific stimuli. In these experiments it must be considered that, owing to the type of study protocol, participants were not blinded to the study medication (resveratrol vs. hydroxytyrosol), so there might have been some placebo effect on the research outcomes since subjects self-limited exercise when they became exhausted. Actually this type of risk was greatly limited by the fact that subjects did not know the experimental hypothesis and did not know the composition of the ingested substances.

Possible rebounds of these results concerning exercise physiology and sports medicine are not negligible. This is due to the fact that all engaged subjects could be considered as semi-professional athletes, and this might be an important matter concerning these experiments. In fact, in athletes who perform intermittent muscle efforts, during the interval between two efforts it is advantageous to deliver as much oxygen as possible to the recovering muscles, thus producing aerobic resynthesis of ATP reserves, and pre-race ingestion of extra virgin olive oil, enriched with hydroxytyrosol, might potentiate this need by enhancing the post effort blood flow in the legs vessels.

\section{Limitations of the Study}

One limitation of this study may arise from the fact that we chose a 2-day interval between the two experimental sessions, and it may be that this time interval was not long enough to wash-out potential effects of the nutraceutical compound assumed in the previous session when, after two days, the subjects assumed the other nutraceutical compound. Indeed, in healthy subjects it was found that after taking $500 \mathrm{mg}$ of resveratrol the half-life of its peak blood concentration was of only 5 hours [28]. On the other hand, hydroxytyrosol blood concentration showed a similar pharmacokinetics in healthy subjects after they had ingested $25 \mathrm{ml}$ of extra virgin olive oil enriched with this nutraceutical compound [29]. Considering the above mentioned experimental results, we are confident that two days could be a long enough period of time to implement the complete wash-out of previously taken nutraceutical compound.

Another limitation of this study may concern some outlier markers which are 
present in Figures 3-5 and, especially for the Vba and thus for Fba, one participant showed excessively high values of these parameters in BRresv, EXresv and EXhydr. This may indicate that the measurements would be highly variable mainly due to the small number of study participants. Therefore, the generalization of our study results needs caution.

In order to overcome these limitations, future experiments with large cohorts of participants and with blood concentration dosage of both resveratrol and hydroxytyrosol during the recovery time after the hand grip exercise, must be programmed.

\section{Conclusion}

In moderately trained subjects, this study shows a post-exercise nearly doubled increase in blood flow of arms peripheral arteries just $60 \mathrm{~min}$ after ingestion of oil enriched with hydroxytyrosol. This physiological adjustment may represent a significant advantage since in the same time span it conveys a larger amount of oxygen to recovering muscles [30]. This condition would be particularly favourable in long-lasting physical performances that exploit aerobic energy sources for the muscles in activity, i.e. long distance races. Thus, hydroxytyrosol could satisfy the growing demand of the multitude of individuals engaged in aerobic performances [31] for adjuvants exclusively based on natural foods.

\section{References}

[1] Wang, Z.M., Chen, Y.C. and Wang, D.P. (2016) Resveratrol, a Natural Antioxidant, Protects Monosodium Iodoacetate-Induced Osteoarthritic Pain in Rats. Biomedicine \& Pharmacotherapy, 83, 763-770. https://doi.org/10.1016/j.biopha.2016.06.050

[2] Ling, K.H., Wan, M.L., El-Nezami, H. and Wang, M. (2016) Protective Capacity of Resveratrol, a Natural Polyphenolic Compound, against Deoxynivalenol-Induced Intestinal Barrier Dysfunction and Bacterial Translocation. Chemical Research in Toxicology, 29, 823833. https://doi.org/10.1021/acs.chemrestox.6b00001

[3] Paradiso, V.M., Di Mattia, C., Giarnetti, M., Chiarini, M., Andrich, L. and Caponio, F. (2016) Antioxidant Behavior of Olive Phenolics in Oil-in-Water Emulsions. Journal of Agricultural and Food Chemistry, 64, 5877-5886. https://doi.org/10.1021/acs.jafc.6b01963

[4] Cetrullo, S., D’Adamo, S., Guidotti, S., Borzì, R.M. and Flamigni, F., (2016) Hydroxytyrosol Prevents Chondrocyte Death under Oxidative Stress by Inducing Autophagy through Sirtuin 1-Dependent and -Independent Mechanisms. Biochimicaet Biophysica Acta, 1860, 1181-1191. https://doi.org/10.1016/j.bbagen.2016.03.002

[5] Leikert, J.F., Räthel, T.R., Wohlfart, P., Cheynier, V., Vollmar, A.M. and Dirsch, V.M. (2002) Red Wine Polyphenols Enhance Endothelial Nitric Oxide Synthase Expression and Subsequent Nitric Oxide Release from Endothelial Cells. Circulation, 106, 1614-1617. https://doi.org/10.1161/01.CIR.0000034445.31543.43

[6] Aarhus, L.L., Vanhoutte, P.M. and Miller, V.M. (1986) Modulation of Endothelium-Dependent Responses by Chronic Alterations of Blood Flow. American Journal of Physiology, 251, H520-H527.

[7] Olesen, S.P., Clapham, D.E. and Davies, P.F. (1988) Haemodynamic Shear Stress Activates a $\mathrm{K}^{+}$Current in Vascular Endothelial Cells. Nature, 331, 168-170. https://doi.org/10.1038/331168a0 
[8] Reyes, J.J., De La Cruz, J.P., Muñoz-Marin, J., Guerrero, A., Lopez-Villodres, J.A., Madrona, A., Espartero, J.L. and Gonzalez-Correa, J.A. (2013) Antiplatelet Effect of New Lipophilic Hydroxytyrosol Alkyl Ether Derivatives in Human Blood. European Journal of Nutrition, 52, 591-599. https://doi.org/10.1007/s00394-012-0361-1

[9] Storniolo, C.E., Roselló-Catafau, J., Pintó, X., Mitjavila, M.T. and Moreno, J.J. (2014) Polyphenol Fraction of Extra Virgin Olive Oil Protects against Endothelial Dysfunction Induced by High Glucose and Free Fatty Acids through Modulation of Nitric Oxide and Endothelin-1. Redox Biology, 2, 971-977. https://doi.org/10.1016/j.redox.2014.07.001

[10] Sriram, K., Laughlin, J.G., Rangamani, P. and Tartakovsky, D.M. (2016) Shear-induced Nitric Oxide Production by Endothelial Cells. Biophysical Journal, 111, 208-221. https://doi.org/10.1016/j.bpj.2016.05.034

[11] Lu, X. and Kassab, G.S. (2015) Integrins Mediate Mechanical Compression-Induced Endothelium-Dependent Vasodilation through Endothelial Nitric Oxide Pathway. The Journal of General Physiology, 146, 221-232. https://doi.org/10.1085/jgp.201411350

[12] Johnson, B.D., Padilla, J. and Wallace, J.P. (2012) The Exercise Dose Affects Oxidative Stress and Brachial Artery Flow-Mediated Dilation in Trained Men. European Journal of Applied Physiology, 112, 33-42. https://doi.org/10.1007/s00421-011-1946-8

[13] Hallmark, R., Patrie, J.T., Liu, Z., Gaesser, G.A. and Barrett, E.J. (2014) The Effect of Exercise Intensity on Endothelial Function in Physically Inactive Lean and Obese Adults. PLoS ONE, 9, e85450. https://doi.org/10.1371/journal.pone.0085450

[14] McClean, C., Harris, R.A., Brown, M., Brown, J.C. and Davison, G.W. (2015) Effects of Exercise Intensity on Postexercise Endothelial Function and Oxidative Stress. Oxidative Medicine and Cellular Longevity, 2015, Article ID: 723679. https://doi.org/10.1155/2015/723679

[15] Paiva, F.M., Vianna, L.C., Fernandes, I.A., Nobrega, A.C. and Lima, R.M. (2016) Effects of Disturbed Blood Flow during Exercise on Endothelial Function: A Time Course Analysis. Brazilian Journal of Medical and Biological Research, 49, e5100. https://doi.org/10.1590/1414-431x20155100

[16] Padilla, J., Harris, R.A. and Wallace, J.P. (2007) Can the Measurement of Brachial Artery Flow-Mediated Dilation Be Applied to the Acute Exercise Model? Cardiovascular Ultrasound, 5, 45. https://doi.org/10.1186/1476-7120-5-45

[17] Tinken, T.M., Thijssen, D.H., Hopkins, N., Dawson, E.A., Cable, N.T. and Green, D.J. (2010) Shear Stress Mediates Endothelial Adaptations to Exercise Training in Humans. Hypertension, 55, 312-318. https://doi.org/10.1161/HYPERTENSIONAHA.109.146282

[18] Krzywinski, M. and Altman, N. (2014) Visualizing Samples with Box Plots. Nature Methods, 11, 119-120. https://doi.org/10.1038/nmeth.2813

[19] Streit, M. and Gehlenborg, N. (2014) Bar Charts and Box Plots. Nature Methods, 11, 117. https://doi.org/10.1038/nmeth.2807

[20] Valls, R.M., Farràs, M., Suárez, M., Fernández-Castillejo, S., Fitó, M., Konstantinidou, V., Fuentes, F., López-Miranda, J., Giralt, M., Covas, M.I., Motilva, M.J. and Solà, R. (2015) Effects of Functional Olive Oil Enriched with Its Own Phenolic Compounds on Endothelial Function in Hypertensive Patients. A Randomised Controlled Trial. Food Chemistry, 167, 30-35. https://doi.org/10.1016/j.foodchem.2014.06.107

[21] Wong, R.H., Howe, P.R., Buckley, J.D., Coates, A.M., Kunz, I. and Berry, N.M. (2011) Acute Resveratrol Supplementation Improves Flow-Mediated Dilatation in Overweight/ Obese Individuals with Mildly Elevated Blood Pressure. Nutrition Metabolism \& Cardiovascular Disease, 21, 851-856. https://doi.org/10.1016/j.numecd.2010.03.003 
[22] Posadino, A.M., Cossu, A., Giordo, R., Zinellu, A., Sotgia, S., Vardeu, A., Nguyen le, H.V., Carru, C. and Pintis, G. (2015) Resveratrol Alters Human Endothelial Cells Redox State and Causes Mitochondrial-Dependent Cell Death. Food and Chemical Toxicology, 78, 10-16. https://doi.org/10.1016/j.fct.2015.01.017

[23] Sader, M.A. and Celermajer, D.S. (2002) Endothelial Function, Vascular Reactivity and Gender Differences in the Cardiovascular System. Cardiovascular Research, 53, 597-604. https://doi.org/10.1016/S0008-6363(01)00473-4

[24] Skaug, E.A., Aspenes, S.T., Oldervoll, L., Morkedal, B., Vatten, L., Wisloff, U. and Ellingsen, O. (2013) Age and Gender Differences of Endothelial Function in 4739 Healthy Adults: The HUNT3 Fitness Study. European Journal of Preventive Cardiology, 20, 531-540. https://doi.org/10.1177/2047487312444234

[25] Green, D.J., Swart, A., Exterkate, A., Naylor, L.H., Black, M.A., Cable, N.T. and Thijssen, D.H. (2010) Impact of Age, Sex and Exercise on Brachial and Popliteal Artery Remodelling in Humans. Atherosclerosis, 210, 525-530.

https://doi.org/10.1016/j.atherosclerosis.2010.01.048

[26] Jensen-Urstad, K. and Johansson, J. (2001) Gender Difference in Age-Related Changes in Vascular Function. Journal of Internal Medicine, 250, 29-36.

https://doi.org/10.1046/j.1365-2796.2001.00843.x

[27] Badrov, M.B., Freeman, S.R., Zokvic, M.A., Millar, P.J. and McGowan, C.L. (2016) Isometric Exercise Training Lowers Resting Blood Pressure and Improves Local Brachial Artery Flow-Mediated Dilation Equally in Men and Women. European Journal of Applied Physiology, 116, 1289-1296. https://doi.org/10.1007/s00421-016-3366-2

[28] Sergides, C., Chirila, M., Silvestro, L., Pitta, D. and Pittas, A. (2016) Bioavailability and Safety Study of Resveratrol 500mg Tablets in Healthy Male and Female Volunteers. Experimental and Therapeutic Medicine, 11, 164-170.

[29] Miro-Casas, E., Covas, M.I., Farre, M., Fito, M., Ortuno, J., Weinbrenner, T., Roset, P. and De La Torre, R. (2003) Hydroxytyrosol Disposition in Humans. Clinical Chemistry, 49, 945-952. https://doi.org/10.1373/49.6.945

[30] Concu, A. (2007) Cardiovascular Adjustments during Exercise: Points and Counterpoints. In: Crisafulli, A. and Concu, A., Eds., New Insight into Cardiovascular Apparatus during Exercise: Physiological and Physio-Pathological Aspects, Transworld Reseach Network, Kerala, 61-83.

[31] Pinna, M., Roberto, S., Milia, R., Marongiu, E., Olla, S., Loi, A., Migliaccio, G.M., Padulo, J., Orlandi, C., Tocco, F., Concu, A. and Crisafulli, A. (2014) Effect of Beetroot Juice Supplementation on Aerobic Response during Swimming. Nutrients, 29, 605-615. https://doi.org/10.3390/nu6020605 
Submit or recommend next manuscript to SCIRP and we will provide best service for you:

Accepting pre-submission inquiries through Email, Facebook, LinkedIn, Twitter, etc.

A wide selection of journals (inclusive of 9 subjects, more than 200 journals)

Providing 24-hour high-quality service

User-friendly online submission system

Fair and swift peer-review system

Efficient typesetting and proofreading procedure

Display of the result of downloads and visits, as well as the number of cited articles

Maximum dissemination of your research work

Submit your manuscript at: http://papersubmission.scirp.org/

Or contact health@scirp.org 